\title{
PITUITARY, PINEAL AND THIRD VENTRICLE TUMOURS
}

\author{
By Joe Pennybacker, M.D., F.R.C.S. \\ The Nuffield Department of Surgery, Radcliffe Infirmary, Oxford
}

The pituitary gland is concerned with such diverse functions as growth, sexual activity, carbohydrate and water metabolism, physical energy and the character and distribution of hair. For its size it is a very eloquent structure and although not solely responsible for any of these activities, it has been described as the leader of the endocrine orchestra. Many of its disturbances of function are more in the province of the physician than the surgeon. This section is concerned only with the effects produced by tumours. For practical purposes only two types need description, the chromophobe and chromophil adenomas. These tumours acquire their descriptive titles from preponderance of either the chromophobe or chromo- phil cells found in the normal gland, although pure growths of either are rare, and in most cases it would be more correct to speak of a mixed-cell adenoma. The basophil element occasionally undergoes hyperplasia and in some cases is associated with what is widely known as Cushing's syndrome, but in about half the recorded cases of this condition the essential lesion seems to be in the suprarenal gland-another example of the inter-relation of the pituitary with other endocrine glands. The basophil adenoma is generally not a surgical problem, and although some good results have followed the implantation of radon seeds in the pituitary gland, treatment usually consists of deep X-rays directed to the region of the sella.

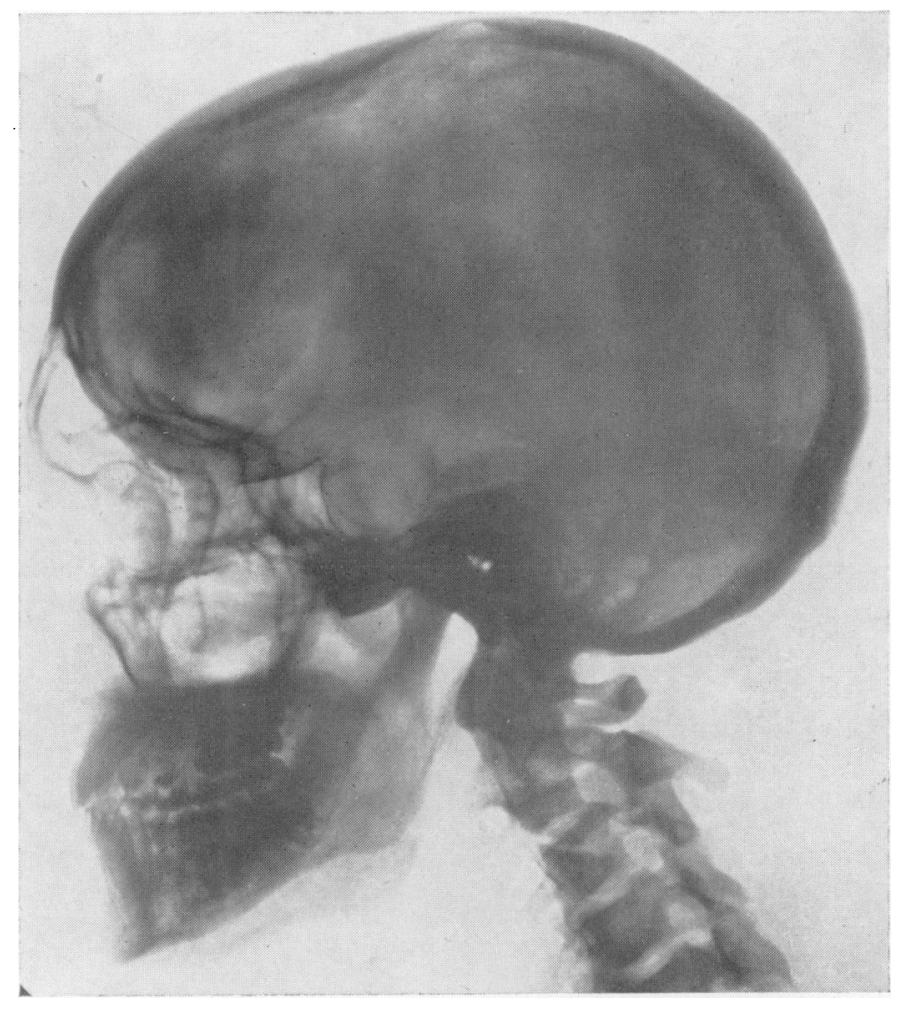

Fig. 1.-Enlarged sella in chromophobe adenoma. 
Although endocrine disturbances are common in pituitary adenomas, the effect on vision is equally important. Indeed, it is generally this which leads the patient to seek advice, and surgical treatment is directed largely to preserving eyesight. The normal gland lies snugly in the pituitary fossa with the optic chiasm suspended above it in a capacious part of the subarachnoid space known as the chiasmatic cistern. As the tumour grows it is easier for it to push the diaphragma sella upwards than to displace the bony walls of the sella, although this does happen eventually and in almost all cases there is radiological evidence of enlargement of the sella turcica by the time the patient comes for treatment (Fig. I). As the tumour expands in an upward direction it first obliterates the chiasmatic cistern, then begins to compress the optic chiasm. The effect of this is to interrupt conduction in the visual fibres coming from the nasal half of each retina which subserve the temporal field of vision:

Bitemporal hemianopia is thus common. It may be complete and easily detectable by simple confrontation tests with the examiner's hands. The patient may be aware of it, and it may be his first symptom. In the early stages of its development, however, the hemianopia may be so slight that it can only be detected by careful perimetry it usually begins with a defect in the upper quade rant of the temporal field, and it is commonly asymmetrical, one eye being more affected than the other (Fig. 2). Rarely the defect may be? limited to one eye; in such cases the tumour preg sumably exerts specific pressure on one part of the chiasm or on the medial border of one optic nerve $\frac{\bar{\rho}}{2}$ although it is often impossible to be certain o\& these relations at operation.

The acuity of vision is not disturbed until the macular fibres are involved and the field defec? encroaches on the fixation point. Thus it is possible for a person to have a complete bitem poral hemianopia, except for a degree or so $\overline{0}$ sparing at the fixation point, and to have norma acuity. As soon as central vision begins to go, the visuai acuity drops quickly, and the field defectu progresses until there is only a bean-shaped island preserved in the nasal field. "Eventually this goes and the eye is rendered completely blind.

Although the initial effect is usually on the optic chiasm, both optic nerves are eventually involved and at operation these nerves are usually found to be splayed apart and flattened on the upper surface of the tumour. This direct pressure induces. primary optic atrophy and some degree of palloro의

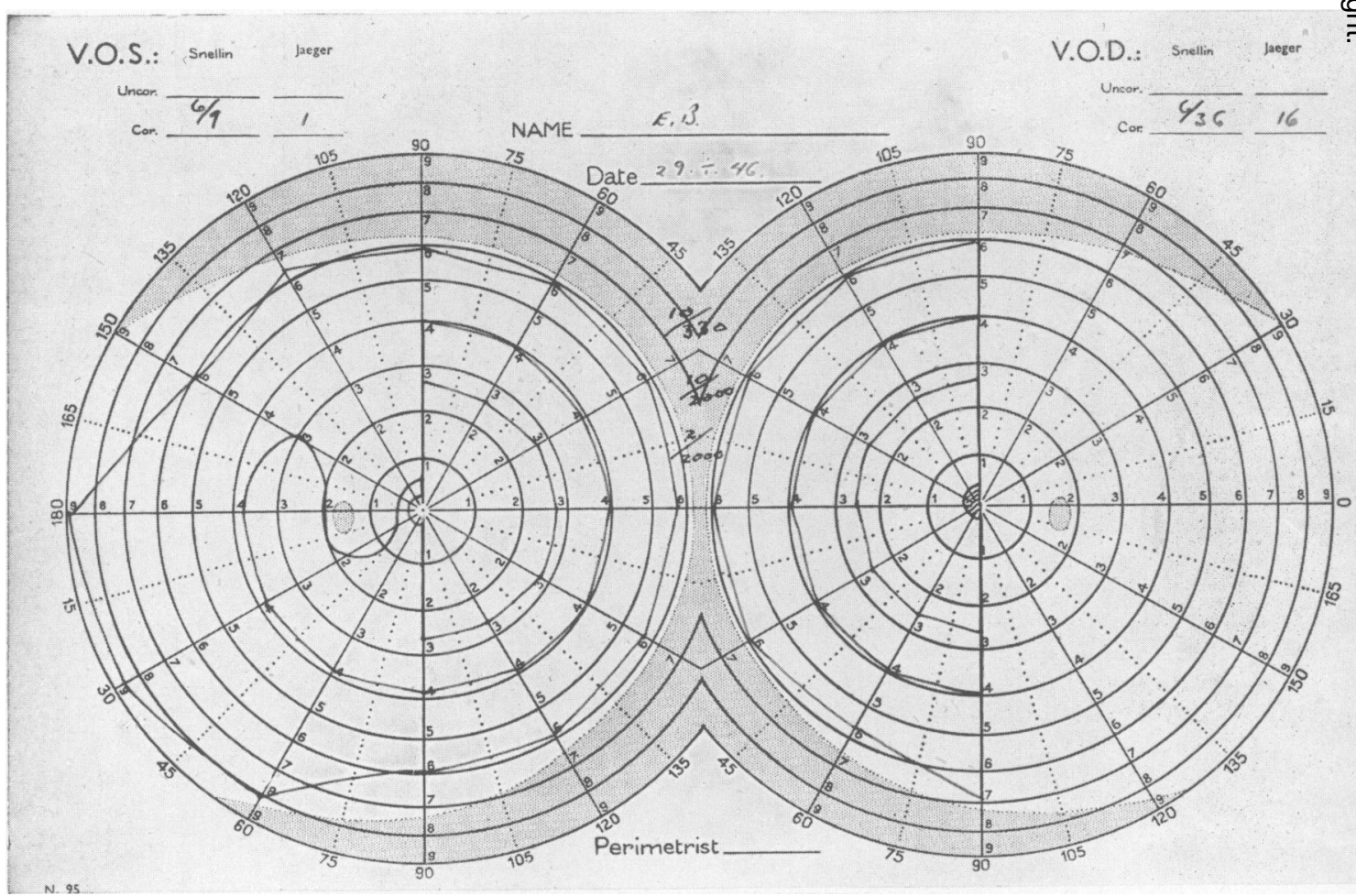

FIG. 2.- Incomplete bitemporal hemianopia in a case of chromophobe adenoma of the pituitary. 


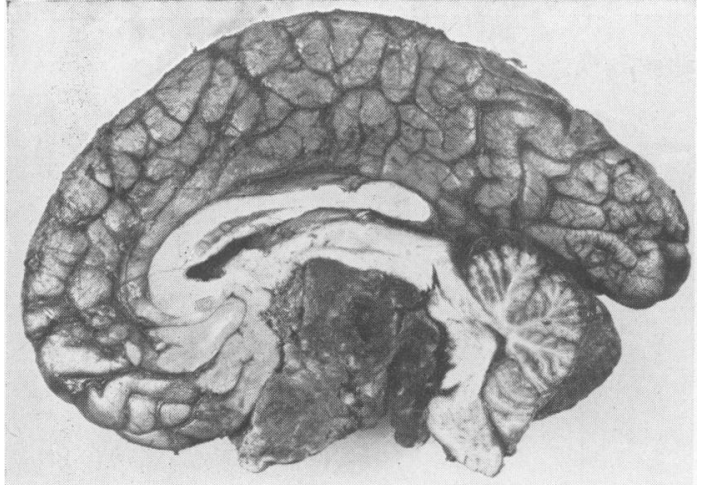

Fig. 3.-Chromophobe adenoma with massive extrasellar extension into third ventricle, compressing brain stem.

the optic discs is usual; in early cases the pallor may be difficult to detect with certainty, and generally compression of the optic nerves and chiasm can be detected earlier and with more certainty by perimetry than by ophthalmoscopic examination.

It is rare for a pituitary adenoma to cause papilloedema. In one of our cases the adenoma had grown laterally to invade the cavernous sinus, and had then burst through this to obstruct the posterior part of the third ventricle and the upper part of the aqueduct of Sylvius. This blockage of the circulation of the cerebrospinal fluid' caused obstructive hydrocephalus, much as any other tumour involving the third ventricle or aqueduct, and gave rise to the common ophthalmoscopic appearance of increased intracranial pressure without any evidence of direct pressure on the optic nerves or chiasm. Although this event is rare, it is common for the tumour to continue to grow upwards and in the late stages to assume a sausage shape, one end resting in the expanded pituitary fossa and the other compressing the anterior end of the third ventricle (Fig. 3). This may also cause a certain amount of obstructive hydrocephalus but by the time it has happened there is usually a well-established primary optic atrophy and papilloedema does not develop.

As mentioned above, the tumour may occasionally expand laterally to involve the cavernous sinus. If so, ocular palsies may result from compression of the third, fourth and sixth cranial nerves as they traverse the sinus. The tumour may burst through the sinus and compress one optic tract, thus producing a homonymous field defect rather than the common bitemporal one. Or it may continue to extend laterally, stripping up the dura from the middle cranial fossa as it does so, and come to expand in the temporal lobe. Similarly, suprasellar extensions may grow to one or other side and form a mass in one frontal lobe rather than in the third ventricle as is usually the case.

Some degree of downward extension is common by expansion and thinning of the bony floor of the sella, but occasionally the floor is eroded completely and the growth then expands to fill the sphenoidal air sinus.

These are the mechanical effects of a pituitary tumour, and as already noted surgical treatment is largely concerned with them. The endocrine disturbances to which they give rise will be discussed in the clinical description of the chromophobe and chromophil adenomas.

Chromophobe Adenomas. These are about twice as common as chromophil tumours. In our material there were 96 verified chromophobe adenomas and 49 chromophil adenomas. They are rare before the age of 20 , but thereafter may occur at ony age. Males and females are about equally affected (52 males and 44 females in our series). It is usually some disturbance of vision which brings them under observation, but by this time there are often some endocrine symptoms as well, and it is not uncommon for a symptom such as amenorrhoea or impotence to lead the patient to seek advice.

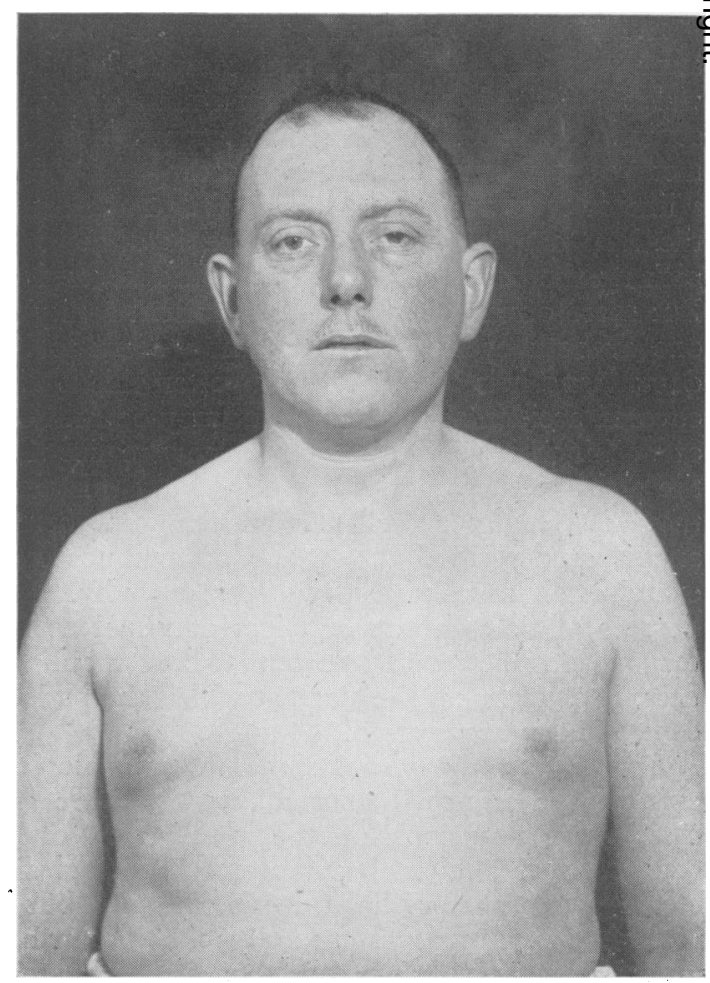

FIG. 4.-Characteristic habitus of a patient with a chromophobe adenoma. 


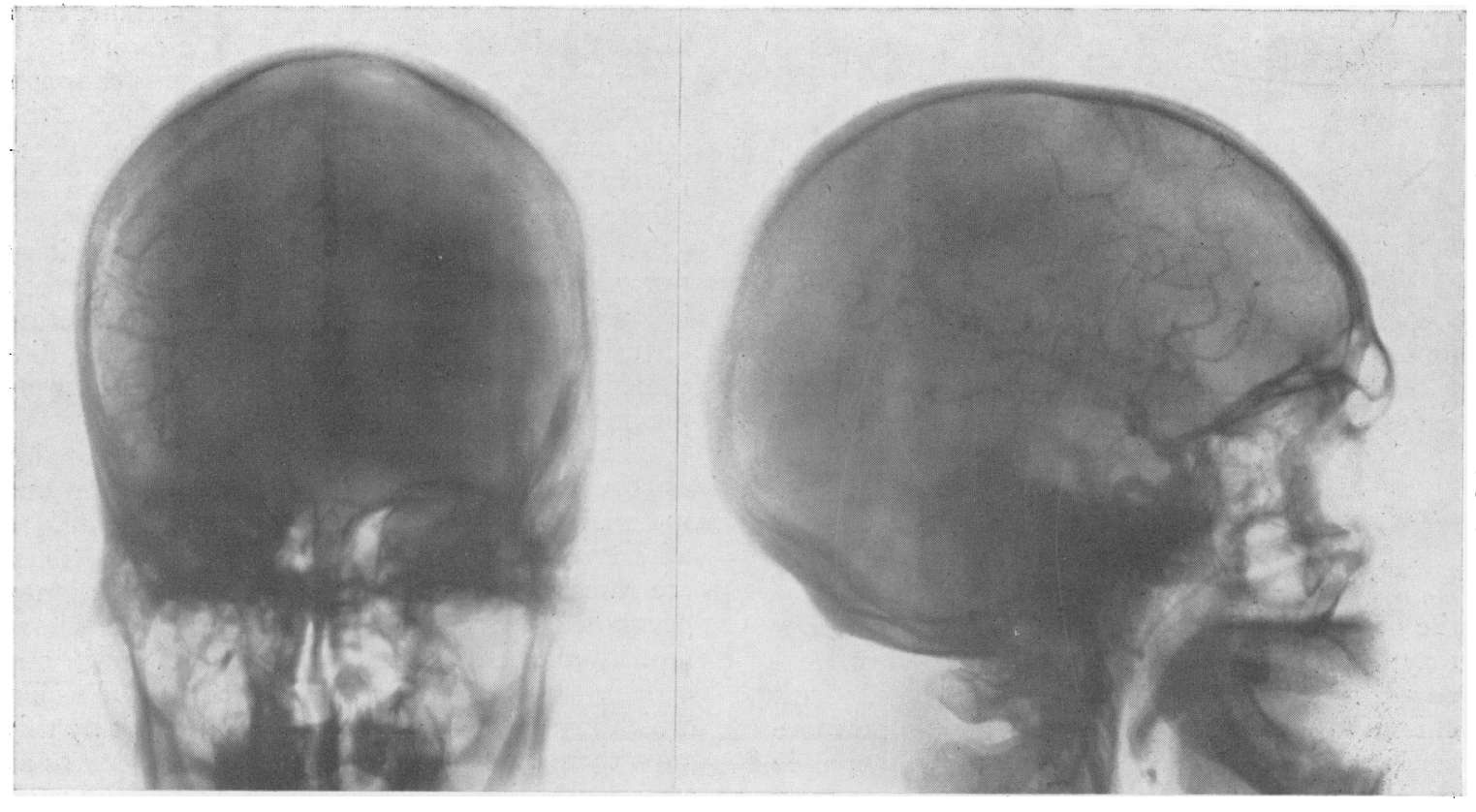

Fig. 5.-Arteriogram in a case of a moderately large chromophobe adenoma. Note displacement of terminal portions of internal carotid arteries.

Intelligent and observant patients may first be aware of a defect in one or both temporal fields of vision before there is any impairment of visual acuity. A defect in the right temporal field for instance may cause difficulty in reading the end of a line of type without ' following it.' A defect in the left temporal field may make it difficult to pick up the beginning of the next line in reading. But more often it is reduction of visual acuity in one or both eyes which takes the patient to his doctor, and it is remarkable how often this is discovered accidentally; the patient may be rubbing or washing his good eye and suddenly realize that the sight in the other is defective. By this time there is usually a well-marked temporal hemianopia as described above, and ophthalmoscopic examination reveals primary optic atrophy. All degrees of field defects and loss of visual acuity may be encountered up to complete bilateral blindness, depending on the stage at which the patient comes under observation.

Apart from the visual signs the neurological examination is usually normal, unless there are extrasellar extensions of the tumour to cause ocular palsies, mental symptoms, hemiparesis, etc. Some degree of headache is common, but it is rarely severe or persistent unless the tumour has grown into the third ventricle to such an extent as to cause hydrocephalus. In such late cases there may be severe and protracted headache, stupor and finally death as from any tumour in volving the third ventricle.

The findings described above are related to the anatomical situation of the tumour. But there are other features, related to endocrine dysfunction, which may be little less striking. The patient is often plump, and in the male the fat is commonly most marked around the lower abdomen and pelvis to produce a somewhat feminine contour. The face is rather round, ascribed by Cushing to prominence of the maxillae (as opposed to the mandible in chromophil tumours) with relative smallness and recession of the chin (Fig. 4). The skin is soft and hairless on the extremities, and the complexion is often somewhat sallow. The hair of the head is soft and silky and that in the axillae and around the pelvis is scanty or may be almost absent. Adult males often need to shave only once or twice a week and to have their hair cut at infrequent intervals, and the pubic hair has the feminine distribution.

Thi se patients often complain of lack of physical energy and of abnormal fatigue in normal occupations. The objective counterparts of these symptoms is sometimes found in low blood pressure readings, lowering of the basal metabolic rate and increased sugar tolerance.

The endocrine symptoms so far described usually manifest themselves in varying degrees; they are often slight and rarely profound. Sexual 


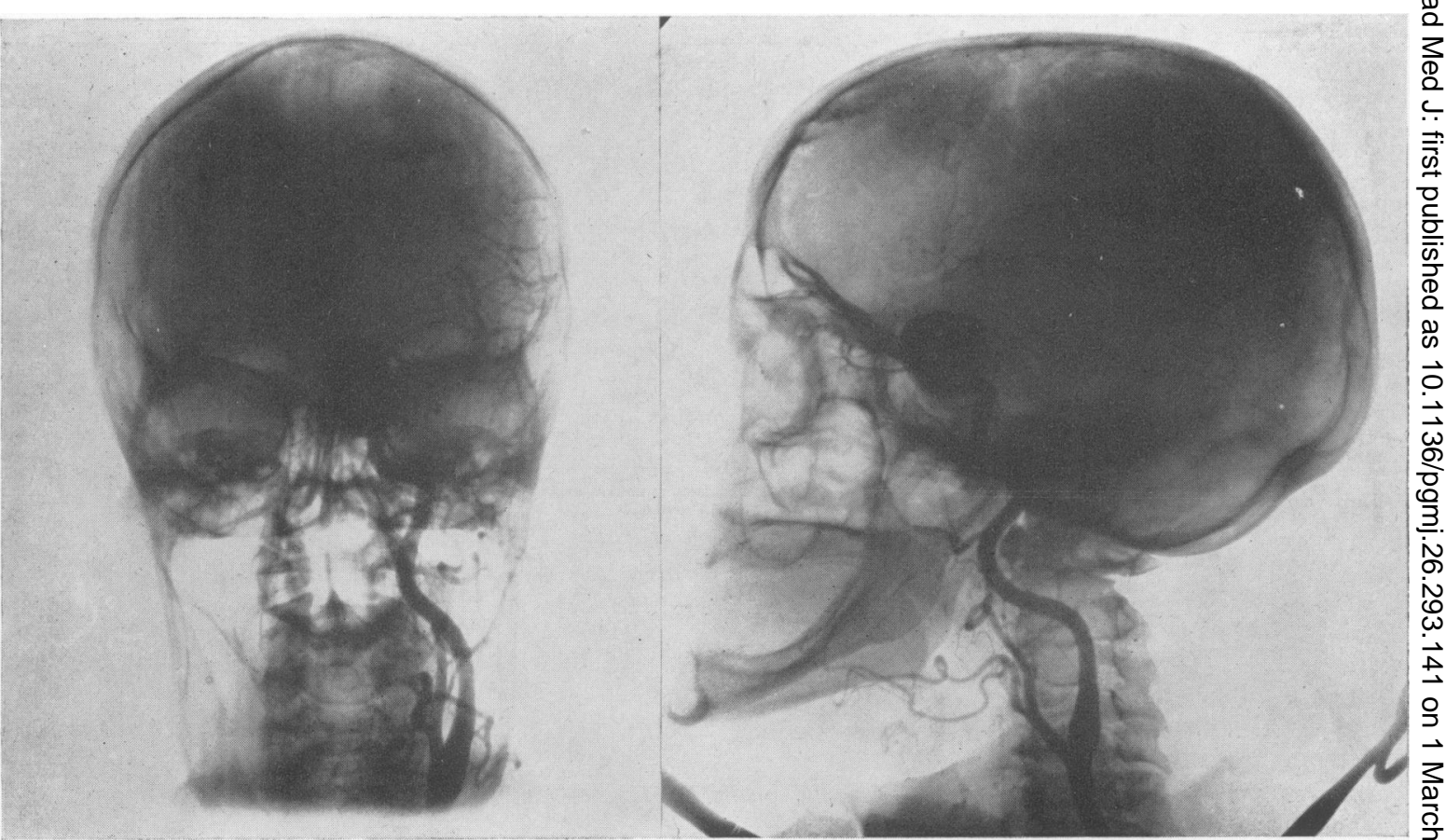

FIG. 6.-Arteriogram of basal aneurysm producing bitemporal remianopia.

activity is usually entirely lost; in females the re is amenorrhoea and sterility, and in males impotence and lack of sexual desire. As the secondary sex characters have usually developed before the tumour starts to grow they are not affected, but in advanced cases there may be some atrophy of the breasts and external genitalia. The control of sexual functions exercised by the pituitary gland is so finely balanced that amenorrhoea or impotence is often the first symptom of a pituitery tumour and may long precede any other symptoms including impairment of vision. It is thus a useful index as to the length of the history. A patient may report a sudden and recent awareness of a bitemporal field defect, but on enquiry prove to have had amenorrhoea for three years. It can be taken that the tumour has been present for at least this length of time.

It should be noted that endocrine symptoms and signs may be largely if not entirely absent, or they may be very specific. Thus occasionally there is a patient with only a bitemporal hemianopia and no other symptoms or signs, especially if the tumour begins to develop late in life when sexual activity has already ceased naturally. Or there may be amenorrhoea or impotence; these indeed are the most constant defects, but occasionally this function too is preserved. We have seen one case of chromophobe adenoma in which there was persistent lactation for two years after child- birth, and this symptom led to the detection of bitemporal hemianopia. Conversely it is very rare to be able to diagnose a chromophobe adenoma from the endocrine manifestations alone, i.e. without a field defect; the same symptoms may be due to various functional disorders of the pituitary. without any enlargement of the sella as determined by skiagrams. By the time the sella is significantly enlarged there is usually a defect in the visual fields, and this means that enlargement of the sella is necessary for the diagnosis of a chromophobe adenoma in almost every case. However there are a few cases of chromophobe adenoma which grow above the diaphragma sellae without enlarging the sella, presumably because they arise from chromophobe cells in the stalk of the hypophysis.

The accessory methods of diagnosis are not often of much help. The cerebrospinal fluid is normal as to pressure and content unless there is a considerable extrasellar extension, in which case the pressure and the protein content may be increased, e.g. to 90-100 mgm. Ventriculography shows no significant abnormality unless the growth has extended to involve the third ventricle and produce ventricular dilatation, or to invade the temporal or frontal lobe and thus cause characteristic deformities. Arteriography likewise reveals little abnormal in early cases, but if there is a massive tumour with extrasellar extension; 


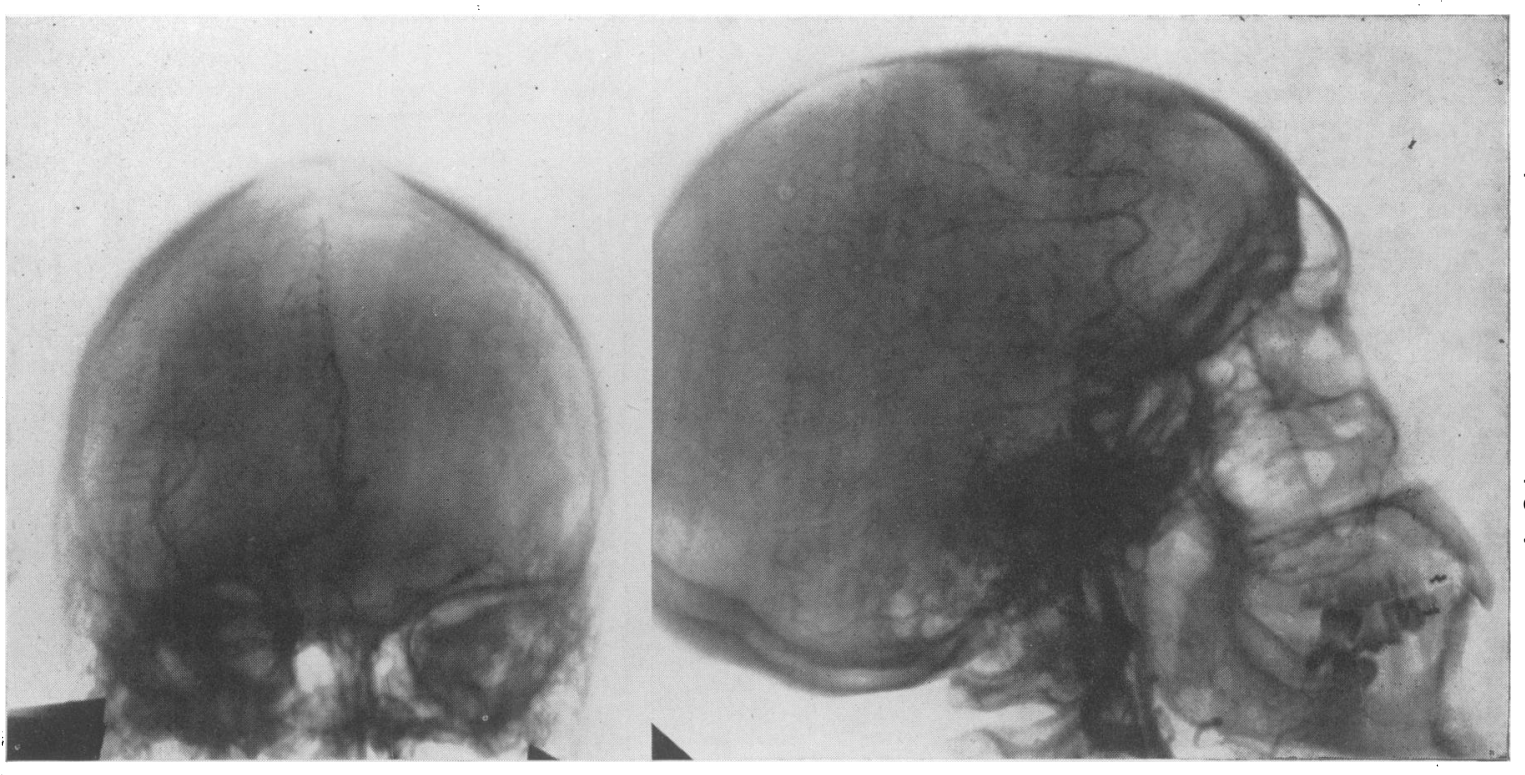

Fig. 7.-Arteriogram of supraseller meningioma. Note abnormal vascular pattern above tuberculum sellae.

there may be characteristic displacements of the terminal portions of the internal carotid and the beginning of the anterior cerebral arteries (Fig. 5).

These accessory diagnostic procedures have a very important role in differential diagnosis. Thus an intracranial aneurysm, e.g. on the anterior communicating or anterior cerebral artery, can cause bitemporal hemianopia (Fig. 6). This lesion usually is associated with a good deal of frontal pain or headache, more than is common with adenomas, endocrine disturbances are slight, and the sella is usually not enlarged. But the diagnosis oan only be made with certainty before operation by demonstrating the aneurysm by arteriography. A more common cause of bitemporal hemianopia is a suprasellar meningioma. This tumour arises from the tuberculum sellae, in front of the chiasm and between the two optic nerves. It grows slowly and may produce a bitemporal or altitudinal hemianopia and even serious reduction of vision before there are any other symptoms and signs, such as those of increased intracranial pressure. There are usually no significant endocrine defects. X-rays show the sella to be of normal size, and indeed the whole skull appears to be normal unless, as is sometimes the case, a hyperostosis can be seen at the site of the tumour attachment to the tumberculum sella. The cerebrospinal fluid pressure is normal, but the fluid commonly contains an excess of protein as the tumour is closely related to the large subarachnoid space in the vicinity of the optic chiasm. The ventriculogram may show little abnormality unless the tumour has attained a sufficient size to displace the frontak horns of the ventricle. An arteriogram, howeveroㅡㅁ may reveal the characteristic vascular pattern of the tumour before it is large enough to produce any major displacement of the anterior cerebral or other arteries at the base of the brain (Fig. 7).

A much more rare cause of chiasmal compression is the suprasellar cholesteatoma or pearly tumour. There is only one example of this tumour in our series, and judging from it the diagnosis may depend almost entirely on exclusion. In this case there was a bitemporal field defect without headache or any endocrine disturbance; normal X-rays, normal cerebrospinal fluid and normal ventriculogram.

More common than any of these diagnostic problems is that of distinguishing a pituitary adenoma from a craniopharyngioma or tumour of Rathke's pouch. The clinical features of this tumour will be described later; suffice it to say now that it is often impossible to make. the differentiation, even at operation, until the tumour has been examined histologically.

Chromophil Adenomas. These tumours declare themselves by producing acromegaly, which may be their only manifestation. Accordingly many of them, the majority indeed, are never verified at operation; but the invariable association of acromegaly with a chromophil adenoma has been too well documented by autopsy records to admit of any doubt. As noted they are less common than 
chromophobe adenomas in hospital practice, possibly because they do not so constantly affect vision. It is also remarkable how stealthily acromegaly may manifest itself; within the family circle or in a small community the change in the patients appearance is so gradual that it may almost be unnoticed.

The physical features are well known, and it is usually the facies rather than the appearance of the extremities which is so striking (Fig. 8). Enlargement and prominence of the frontal sinuses causes an overhanging brow, the nose is enlarged and the lower jaw juts forward so that the lower teeth rest in front of the upper ones in biting. Enlargement of the mandible gives rise to wide spacing of the teeth which, against the large background of the jaw and tongue, appear to be smaller than normal. The tongue is broad and thick and deeply furrowed. The scalp likewise is thick and furrowed and the hair is coarse and wiry. The affection derives its name from the enlargement of the extremities, and there is usually enlargement of the hands and feet so that a woman finds that her rings are having to be expanded and larger sizes in gloves and shoes worn. The soft tissues alter so that the hand feels like a cushion, and it has been said that this feeling is characteristic enough to enable a physician to recognize an acromegalic by shaking hands with him in the dark. The trunk and limbs are generally somewhat massive with coarse, hairy skin. There is often a considerable thoracic kyphosis which gives rise to a deep thoracic cage and may account for some loss of bodily height. The voice becomes deep and somewhat thick because of enlargement of the air sinuses, tongue and vocal cords.

This description of course applies to wellmarked cases.' There are many others in which only a few features will be obvious, and some in which the changes are slight enough to give rise to the term ' fugitive acromegaly.' In the definite cases there is almost always enlargement of the sella turcica as seen in skiagrams, indicating expansion by a tumour (Fig. 9). It is a remarkable fact, however, that the tumour does not often expand sufficiently to compress the optic chiasm and endanger eyesight; only three of 49 cases required operation for preservation of vision whereas with chromophobe adenomas necessity for operation is the rule. It is also a fact that acromegaly can progress up to a point slowly over many years and then seemingly become arrested.

If the tumour begins in adolescence before the epiphyses of the long bones have united, the bones continue to lengthen and gigantism results. The acromegalic giant is familiar in medical literature, but on the whole marked degrees of gigantism are rare. An incongruous feature is that the giants are often physical weaklings, and this applies to some extent to acromegalics in general; their massive build and features give an appearance of great physical strength which is not borne out by trial. Indeed the patient often comes to his doctor complaining of weakness and fatigue. In some there may be diabetes mellitus, ? not infrequent accompaniment of acromegaly. It has been said that diabetes is the commonest cause of death in acromegaly but this has not been the case in our experience; we have had more with severe degrees of arterial hypertension than with diabetes, and it may be the headache due to high blood pressure which brings the patient to his doctor.

Another type of headache, not associated with hypertension, is a common and sometimes a very distressing symptom. It is often described as a tight or bursting feeling between the temples, and may be continuous for weeks or months with little variation and may be associated with some lachrymation. It is clearly not due to increased intracranial pressure as there is no papilloedema

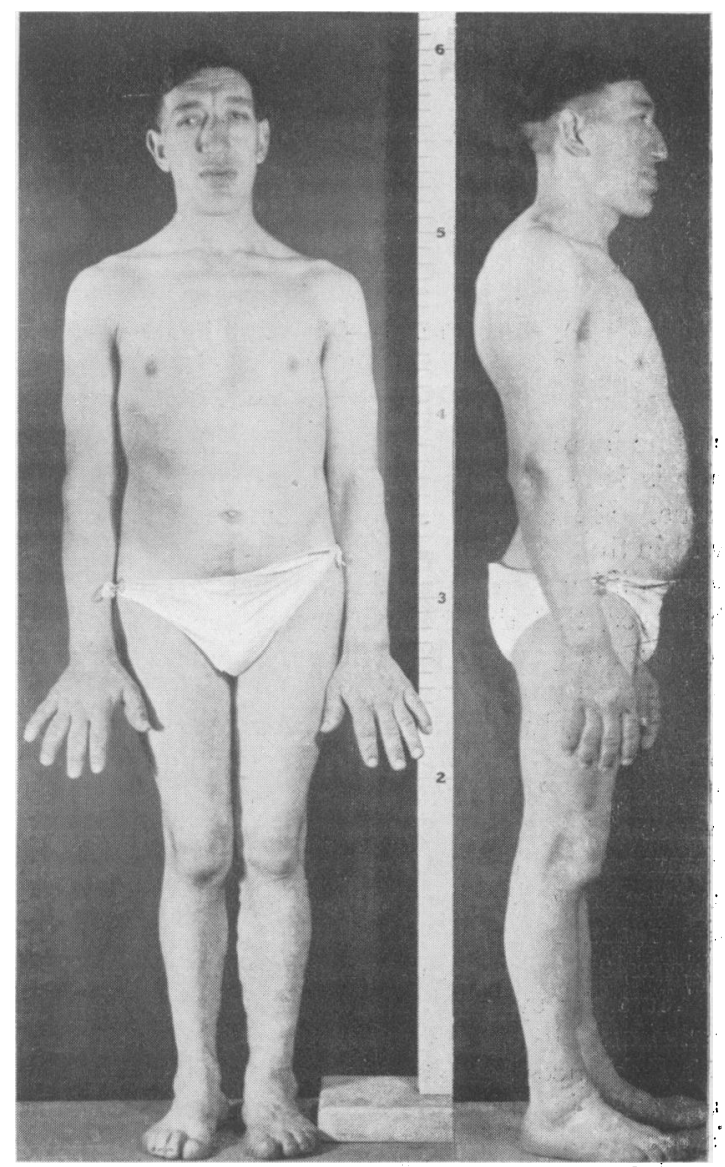

FIG. 8.-Acromegaly. 


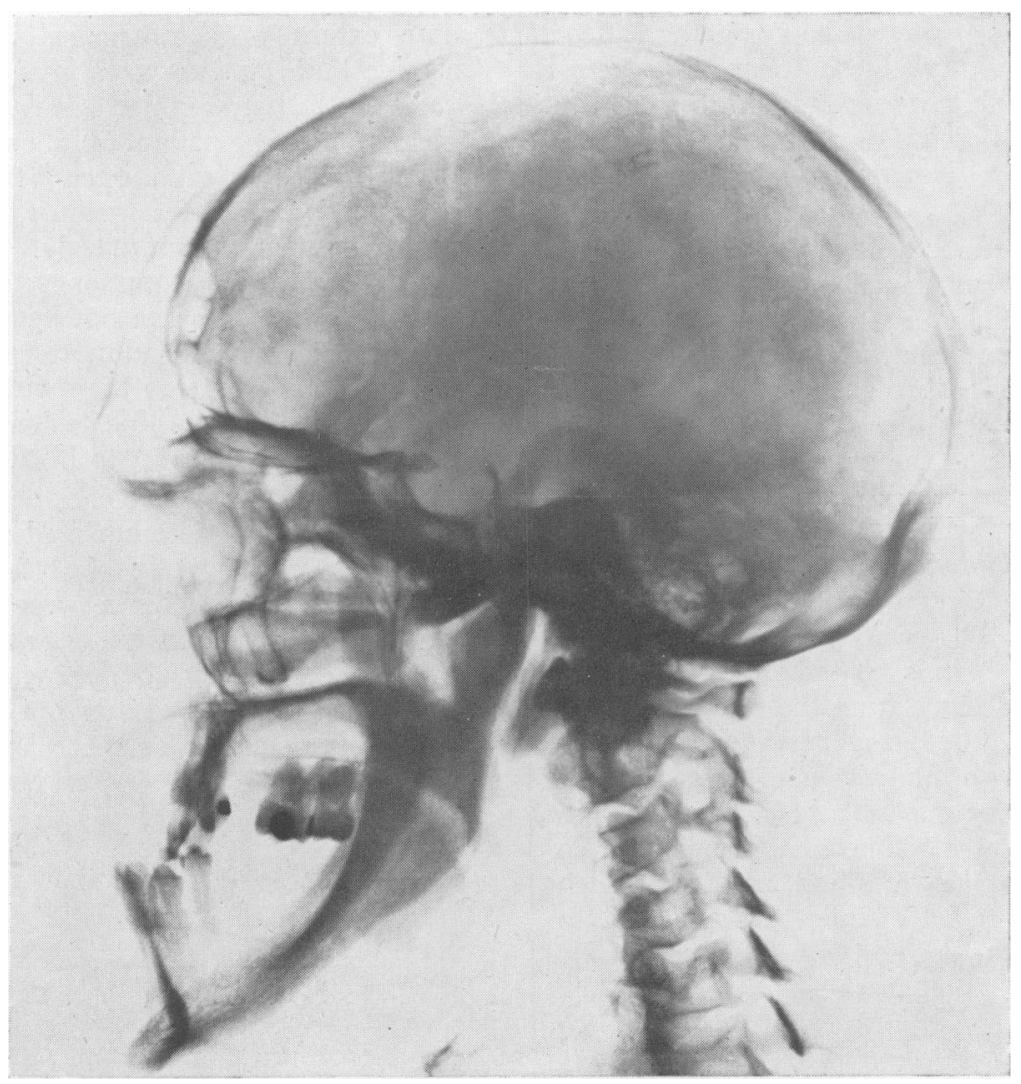

Fig. 9. - The skull in acromegaly. Note large frontal sinuses, enlarged sella, malocclusion of teeth, and prominent lower jaw.

and no increase in the cerebrospinal fluid pressure, nor is it at all relieved by decompressing operations. It has been ascribed to increased pressure within the sella itself, but it is a matter in which it is very difficult to determine causes. It is a fact, however, that this headache may eventually clear up spontaneously, and that it usually responds satisfactorily to X-ray therapy.

Acromegaly is usually accompanied by depression or abolition of sexual function although this feature is not as constant as in the chromophobe adenomas. In females galactorrhoea is common, and the disease may manifest itself after a pregnancy. Enlargement of the thyroid sometimes occurs and a large goitre may cause considerable respiratory embarrassment.

Thus the symptoms of a chromophil adenoma are similar to those of the chromophobe tumour, the differences being that in acromegaly the skeletal and somatic changes are more marked, headache is more common and serious visual disturbances are less common. There are, of course, many cases in which some features of both types of tumour are manifest, i.e. the patient with most of $\frac{3}{\sigma}$ the features of a chromophobe adenoma who has: acromegalic hands and feet, and histologically it may be difficult to classify such ' mixed tumours ' as either chromophobe or chromophil.

As to the clinical course of these tumours the chromophobe adenoma usually progresses steadily, $\delta$ unless it is treated, to cause blindness and later $₹$ death by developing a large extrasellar extension.은 e.g. in the third ventricle. This may take several years, or a much shorter time depending on how? rapidly the tumour grows. The untreated chromo-n phil adenoma on the other hand carries a longer expectation of life, and many patients with acro- $N$ megaly beginning in early adult life live to the fifth and sixth decades. As noted above, the acro-0 megalic may die from hypertension or diabetes and those cases in which asthenia is a marked feature succumb to relatively mild infections.

Treatment. For the chromophobe adenomatat operation is usually called for to preserve vision. It should be stressed that removal of a pituitary tumour can hold out little hope of improving the 


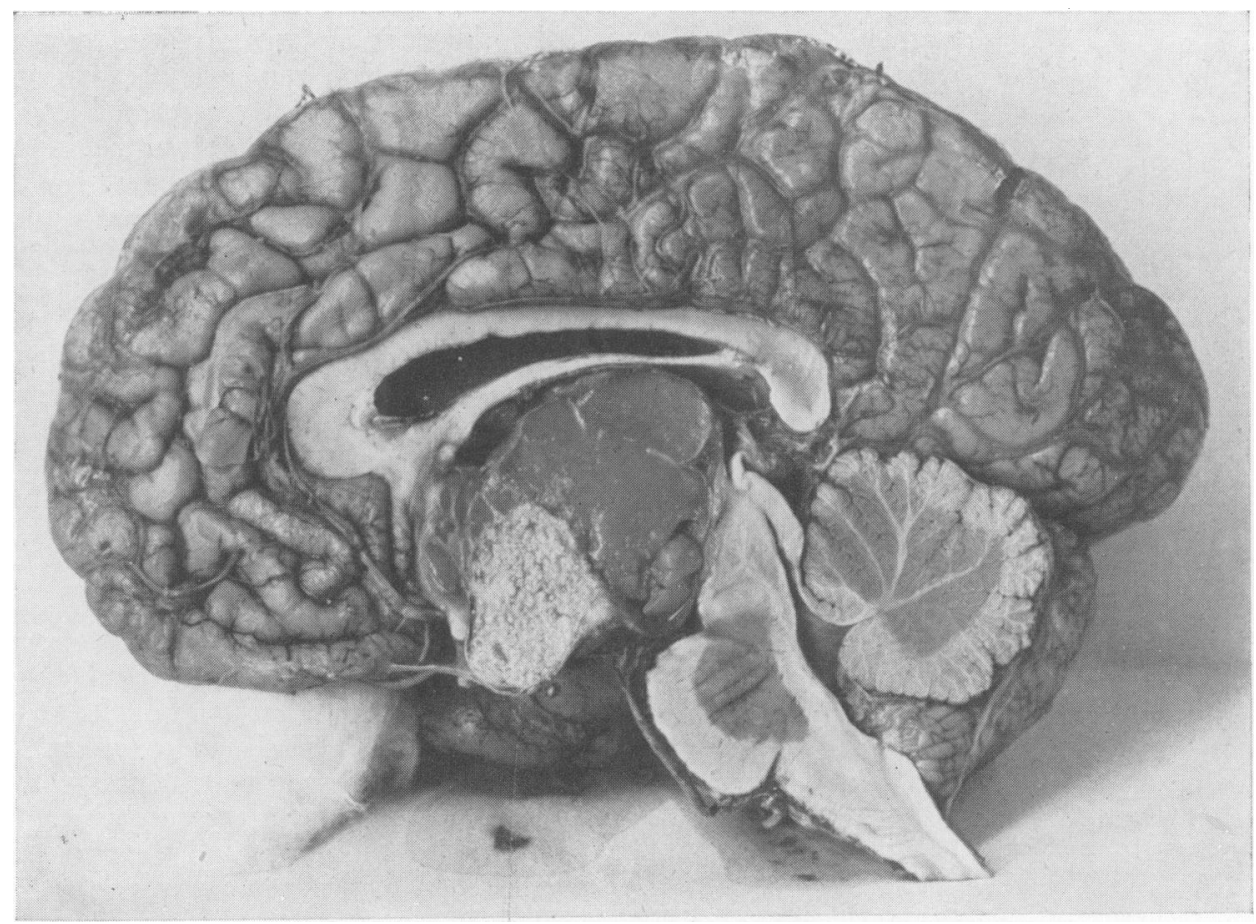

FIG. I0.-Rathke Pouch Tumour. Note calcification in lower part and cyst filling third ventricle.

endocrine symptoms, e.g. sterility, asthenia and the like. Although the patient often expresses some improvement in general well-being, it is difficult to say how much of this is referable to improvement or restoration of vision. As to the time to operate, the indication generally is evidence of a progressive field defect, even before central vision is affected. Within limits, operation offers the best chances in early cases with incomplete field defects and no loss of central vision; in such the fields may return to normal after operation. Further, the more acute and recent the loss, either of fields or acuity of vision, the better chance there is of restoration by operation. Thus in one case under observation with a slight bitemporal defect there was a rapid loss of vision in both eyes within a few days to almost complete blindness. Operation undertaken as an emergency disclosed a cyst in the tumour, evacuation of which restored central vision at once and within two weeks the fields were virtually full again. On the other hand, longstanding cases of bitemporal hemianopia, with perhaps considerable loss of acuity in one eye, often do not improve much after operation, but the operation should prevent further deterioration which would otherwise be inevitable.

The operation commonly practised nowadays entails an osteoplastic craniotomy in the frontal region. The frontal lobe is elevated from the anterior fossa to expose the optic nerve and chiasm, and the tumour usually presents as a smooth purple or pinkish dome between the two optic nerves and in front of the chiasm. These tumours are often cystic and a needle is inserted for a trial aspiration. The capsule is then incised and the interior of the tumour is removed by suction, special biting forceps and curettage. The tumour tissue is usually soft and is easily dealt with by these means. The collapsed capsule is then gently dislodged from the optic nerves and chiasm and as much as possible is excised. Bleeding from the

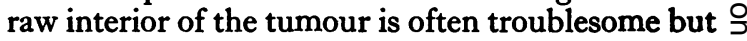
it can usually be checked by firm pressure with $D$ cotton wool pledgets, or by the application of fibrin foam or similar haemostatic agents. As soon as the $N$ cavity is dry, the bone flap is replaced and sutured in position and the scalp incision is closed with a double layer of interrupted stitches.

It will be seen from this brief description that the tumour is not completely removed. To do so would often entail total removal of all the active pituitary gland, and would produce a state of profound pituitary cachexia as in a hypophysectomized animal. In humans this state may be fatal, so it is just as important not to remove too much as to remove sufficient to provide freedom for the 


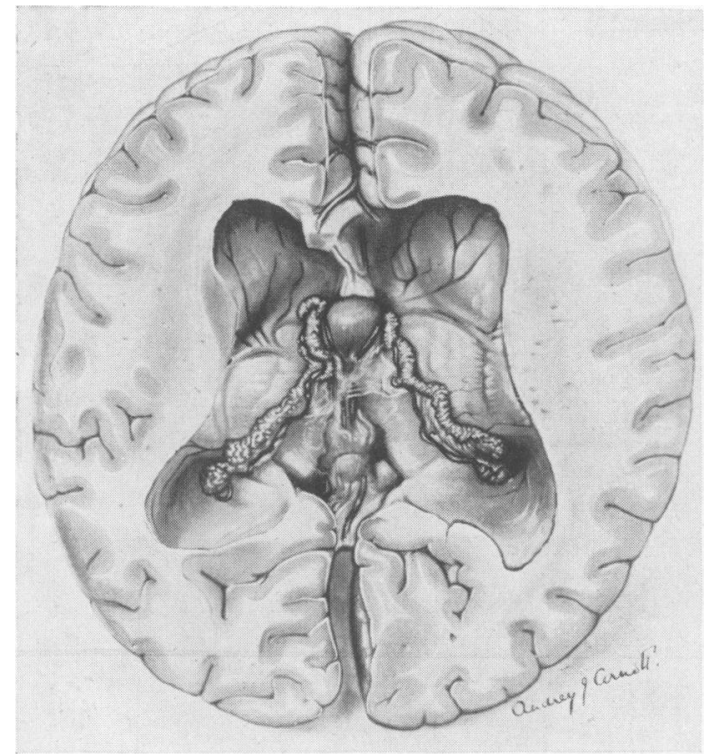

Fig. 12.-Colloid Cyst of Third Ventricle. The roof of the third ventricle has been removed to show the cyst impacted in the foramen of Monro.

optic nerves and chiasm, which is the primary purpose of the operation. As some neoplastic tissue is left, recurrence of visual symptoms may be expected, but a satisfactory operation may provide relief for 10 or 12 years or even longer. There is a considerable body of evidence that recurrence may be delayed by X-ray treatment directed to the sella after operation, and this is now the rule in many surgical clinics. We think it unwise to irradiate these tumours before operation, because occasionally the radiation causes the tumour to swell during the course of treatment and further damage may be done to the visual pathway. Thus we have seen a case of acromegaly without any field defect develop a complete bitemporal hemianopia while undergoing radiation treatment for the type of headache mentioned above.

The same type of operation is employed in acromegaly when there is evidence of progressive chiasmal compression endangering eyesight, but as already mentioned, this is rare. The operation is more tedious because of the altered architecture of the skull, and some surgeons prefer the transsphenoidal approach. For this an incision is made in the mucous membrane of the upper gum beneath the nose and a submucous resection of the septum carried back to the sphenoidal sinus. In some cases of large tumours of both types, the floor of the sella is eroded so that the tumour presents in the sinus and can be excavated much as from above by the transfrontal approach. In others the thin bony floor of the sella has to be re- moved to expose the tumour. This operation is said to be safer in cases of very large tumours in which little vision remains, because of the vulnerability of the greatly stretched and flattened optic nerves to even gentle manipulations when the tumour is approached from above.

The endocrine deficiencies are generally not benefited by operation. Indeed, as mentioned above, they may be more marked after an extensive operation. For them some form of substitution treatment is worth trying but the results are rarely dramatic. Anterior pituitary hormones sometimes help, and testosterone given by mouth or implantation sometimes restores sexual activity in males and increases general well-being and physical strength.

\section{Rathke Pouch Tumours}

These tumours, also spoken of as craniopharyngiomas, adamantinoma and cranio-buccal cysts develop from a remnant of the foetal craniobuccal pouch. This is an outgrowth from the primitive stomodaeum which fuses with a downward evagination from the forebrain. From the latter the infundibulum and the pars posterior of the pituitary gland develop, while the buccal outgrowth becomes sealed off from the enteric canal and is responsible for the pars anterior and pars intermedia of the pituitary. A part of the buccal pouch may be isolated during development and later begin to develop a cyst, or to show neoplastic properties. These lesions are usually cyştic; develop anterior to the pituitary gland and are commonly above the diaphragma sella; thus they are often called suprasellar cysts. The anatomical and physiological effects of such a tumour are thus very similar to those produced by pituitary adenomata, but there are important differences:

Being the result of developmental abnormalities, it is not surprising that the majority of them cause symptoms in early childhood or adolescence, although they may manifest themselves at any age and we have had patients over 60 years of age with recent symptoms of such tumours. The local or anatomical effects are generally one of two types:- (I) The tumour may grow in such a way that it compresses the chiasm and later one or both optic nerves to cause bitemporal hemianopia, unilateral or bilateral blindness, with primary optic atrophy. In this group the ophthalmic picture is in no way different from that produced by pituitary adenomas. (2) Or the tumour may grow straight upward to fill the third ventricle from below, without directly affecting the visual pathway (Fig. 10). In this group the clinical picture is one of increased intracranial pressure which results from obstruction of the foramen of Monro and the aqueduct of Sylvius. There is severe headache, 


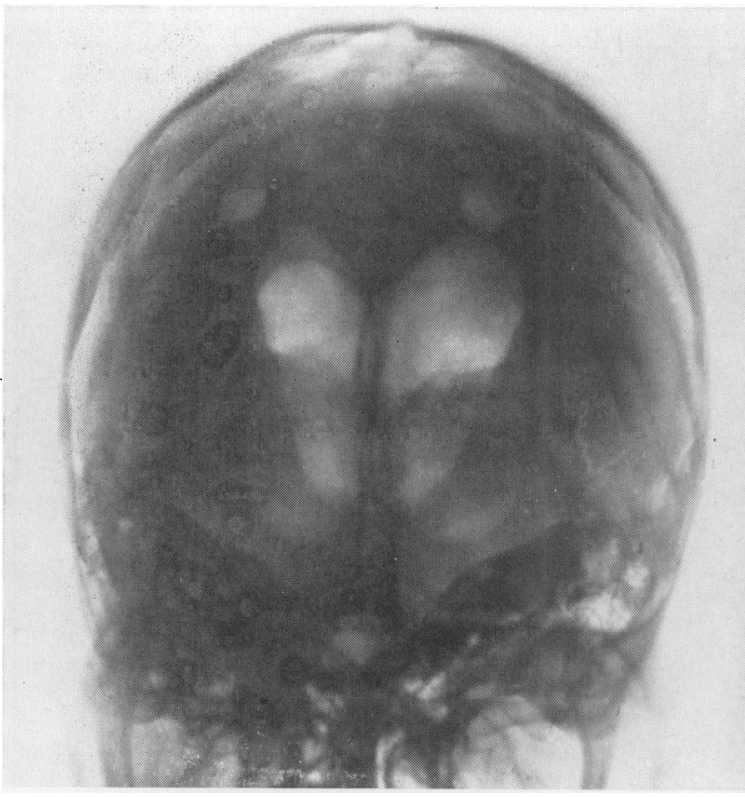

Fig. 13.-Ventriculogram of colloid cyst of third ventricle. Note spherical filling defect below and behind foramen of Monro.

vomiting, papilloedema and later secondary optic atrophy, and these tumours cause death from increased intracranial pressure much as with any other tumour of the third ventricle.

The endocrine effects seem to bear no relation to the anatomical disposition of the tumour; in both types there is usually marked hypopituitarism. In childhood there is often some degree of graceful dwarfism, and the Lorain type is characteristic. Sexual development is delayed and imperfect. In later adult life there is often a smooth, hairless skin on the trunk and limbs, silky hair on the head and amenorrhoea and impotence as seen in pituitary adenomas.

The X-ray appearances may be characteristic. The sella is often not significantly enlarged, but in the majority of cases some calcification is apparent in the vicinity of the sella. This appearance is almost pathognomonic (Fig. II). If the cyst has grown up into the third ventricle there may also be appearances due to increased intracranial pressure, namely convolutional thinning of the vault of the skull and, in children, separation of the sutures.

It will be seen that the diagnosis may be easy if a hypopituitary child or adult presents with a bitemporal field defect and radiological evidence of calcification in the sellar region. But there are some cases of adults with a chiasmal syndrome and no clear radiological abnormalities; in such the absence of enlargement of the sella is unlike a pituitary adenoma, and the hypopituitarism is un-

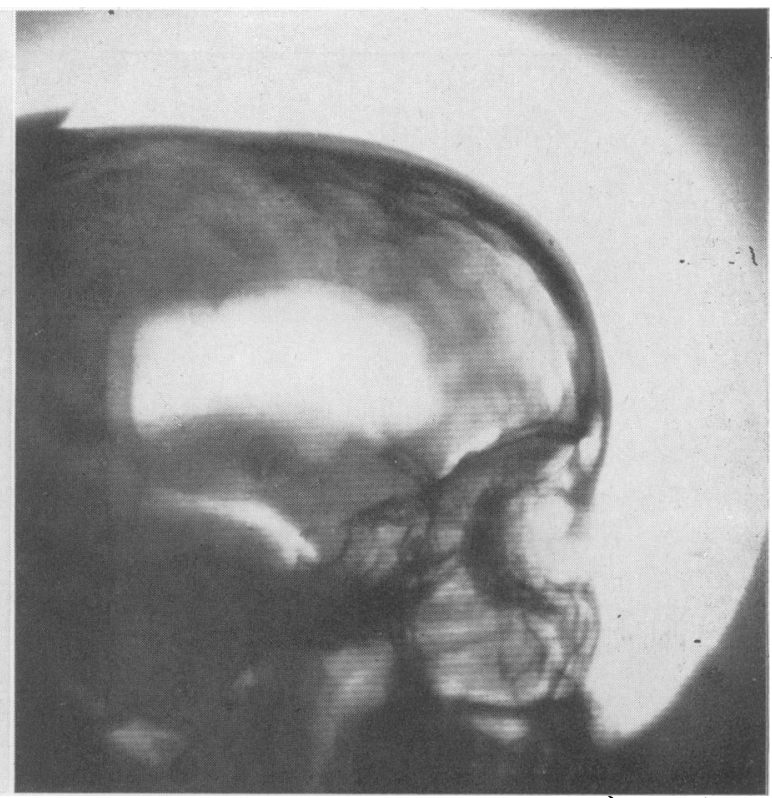

like a suprasellar meningioma, but in some of these the pathological diagnosis can only be made at operation.

Treatment. It must be admitted that these tumours are generally not very favourable for surgical treatment. Those that involve the chiasm are often so adherent to neighbouring structures that they cannot be removed completely, and this is even more true of those growing up into the third ventricle. As mentioned, they are often cystic and the operation usually amounts to emptying the cyst and removing as much of its wall as possible, though in a few cases it is possible to remove the whole tumour successfully. For the cases presenting with chiasmal symptoms, this entails much the same sort of operation as for pituitary adenomas, i.e. by elevation of the frontal out there may be a period of relief for several years but recurrence is the rule. Simple aspiration of the cyst is rarely effective for longer than a few weeks.

For the tumours causing obstruction in the third ventricle there are two types of operation. In the first, the tumour is exposed by excising a window of the frontal lobe to open into the anterior horn of the ventricle (Fig. I4). The tumour can then be seen impacted in the foramen of Monro. More room can then be obtained if the third ventricle is opened by cutting the anterior pillar of the fornix, as well as by aspirating the cyst. It is then often possible to remove a large part of the cyst wall, but attempts to remove the solid part of lobe, and if an extensive removal can be carried 

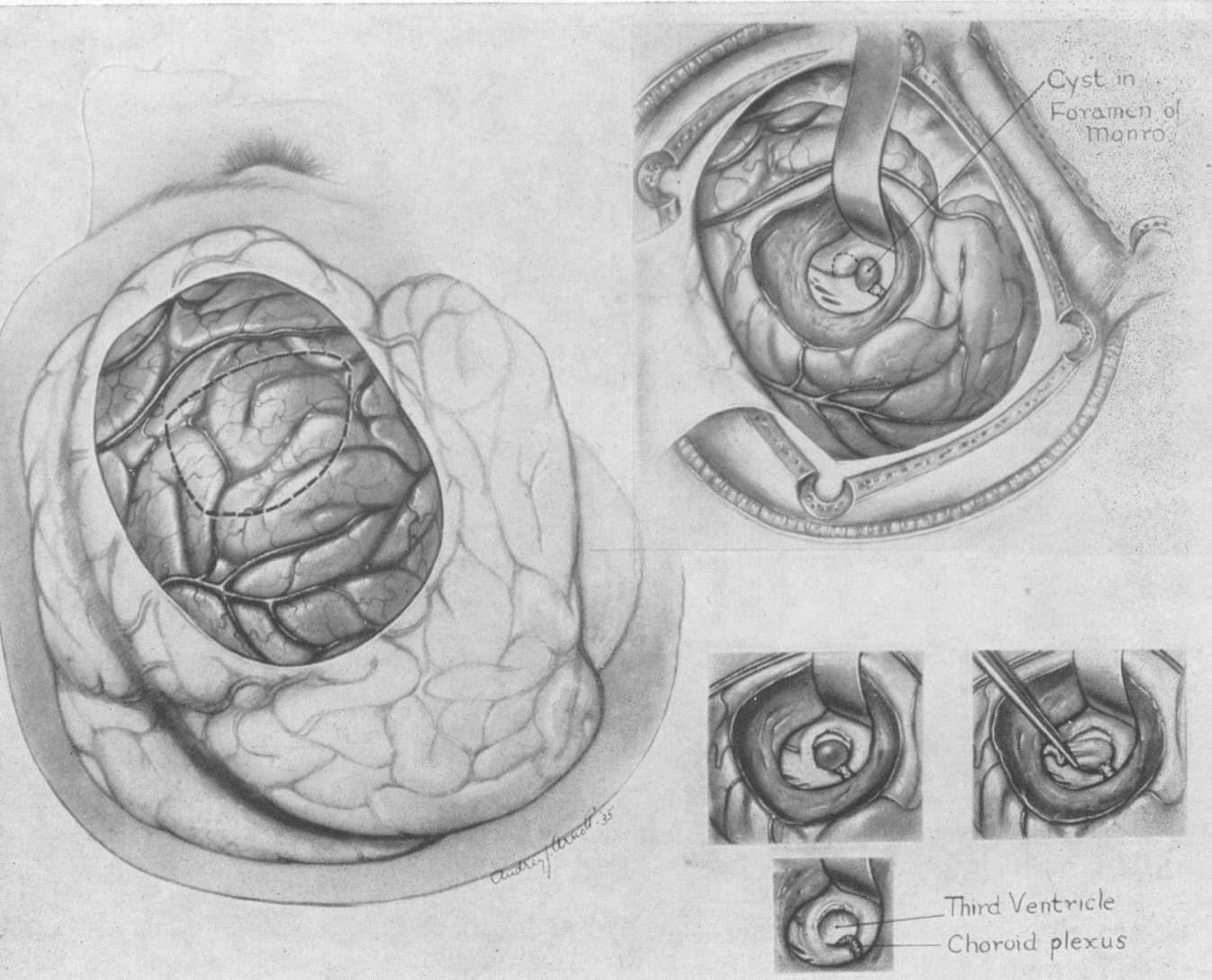

FIG. 14.-Transventricular removal of colloid cyst in third ventricle.

the tumour in the floor of the third ventricle are usually unsuccessful and carry a high mortality from a profound hypothalamic disturbance in the immediate postoperative period.

More recently attempts have been made to deal with this type of tumour by ventriculocisternostomy, an ingenious procedure devised by Torkildsen. In this operation a rubber or polythene tube is passed from the lateral ventricle to the cisterna magna so that the cerebrospinal fluid does not have to traverse the third ventricle, aqueduct and fourth ventricle. This procedure will deal with the problem of increased intracranial pressure, but it may not relieve the local effects of pressure exerted on the third ventricle by the cyst. In other words headache and vomiting may cease and papilloedema subside, and yet the patient remains drowsy, apathetic and in a state of lowered vitality. In some cases, however, the effect is strikingly beneficial and on the whole this procedure seems to hold out more promise than attempts at radical transventricular removal.

Because of the unsatisfactory results of operation in Rathke pouch tumours, there have been many attempts to deal with them by X-ray therapy, but in our series we have no good evidence that they are radio-sensitive.

\section{Third Ventricle Tumours}

The commonest type of third ventricle tumour is a glioma growing from one of its walls, e.g. the optic thalamus. Much rarer are the benign $\omega$ tumours arising from the blood vessels, meninges $\sigma$ and ependyma. Thus angioma, meningioma and $\varphi$ ependymoma have all been encountered, but $\mathbb{\Phi}$ commoner than any of them is what is known as a ? colloid cyst. This is a spherical cyst containing mucoid viscous fluid which develops from and is $\frac{O}{D}$ adherent to the inferior surface of the roof of the third ventricle just behind the foramen of Monro. 

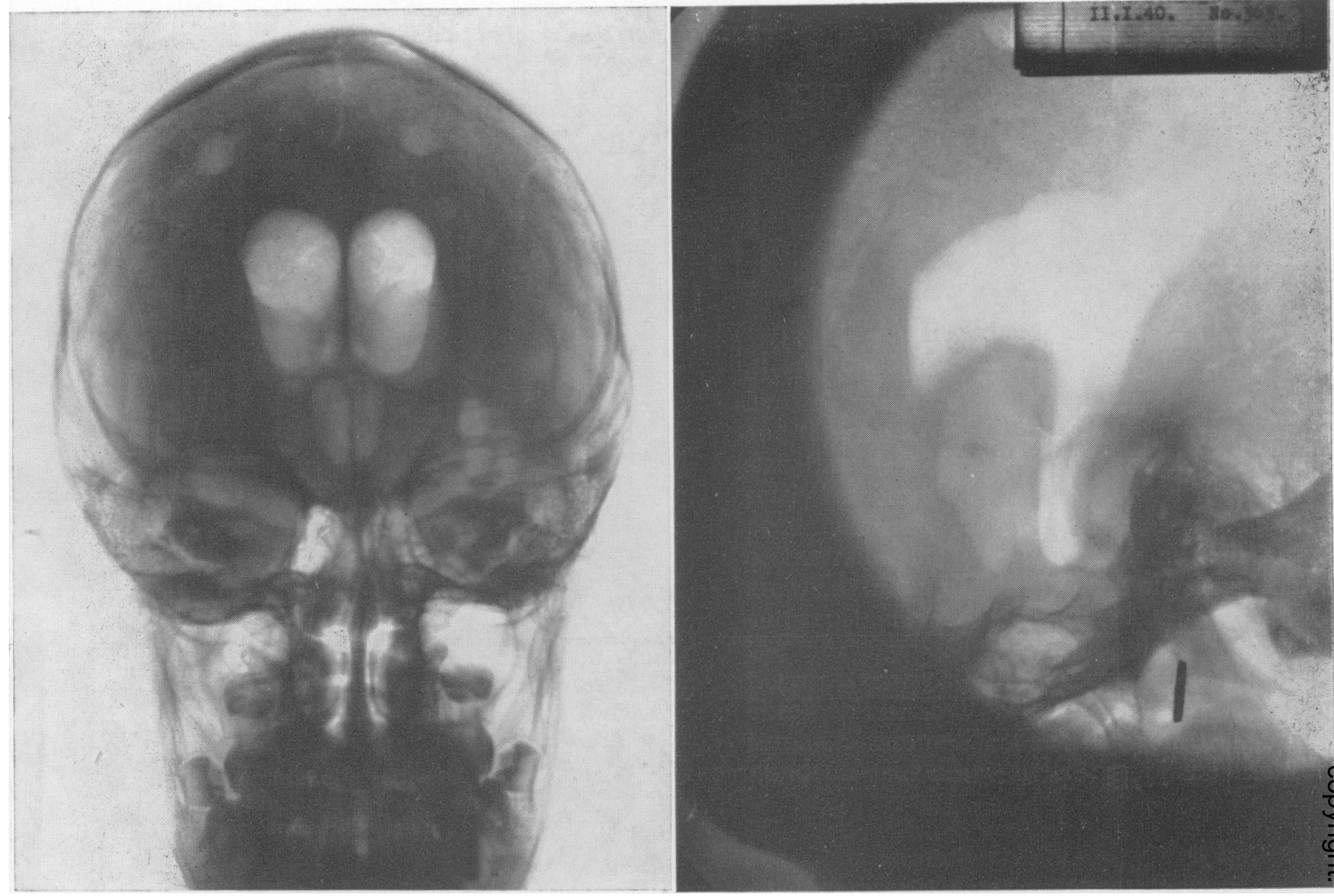

FIg. I 5.-Ventriculogram of pineal tumour. Note rounded filling defect in postero-superior part of third ventricle.

It may attain a size of 2 to $3 \mathrm{~cm}$. in diameter and it plugs up both foramina and causes hydrocephalus much as does a Rathke pouch cyst (Fig. I2).

Third ventricle tumours cause symptoms of increased intracranial pressure and there may be little or nothing in the way of focal neurological abnormalities. Because of their situation they may occasionally act like a ball-valve and produce paroxysmal headache, vomiting and stupor, but this is by no means characteristic. There are occasionally symptoms of hypothalamic disorders, e.g. somnolence, obesity, abnormalities of water metabolism, but these too may occur as indirect effects of a lesion elsewhere, e.g. in the fourth ventricle and are rarely diagnostic.

The diagnosis of a third ventricle tumour rests in ventriculography, and the appearances are generally unmistakable (Fig. I3). The gliomas cannot be removed and treatment usually consists of a ventriculocisternostomy (v.s.) followed by $\mathrm{X}$-ray therapy. The intrinsic tumours call for an exploration and this is usually done by the transventricular route described above (Fig. 14). Many of them can be completely removed with full recovery. Those which cannot be removed should have a ventriculocisternostomy and radiation subsequently if necessary, e.g. in extensive angiomas.

\section{Pineal Tumours}

Tumours of the pineal gland (Fig. I6) are rare, but they produce effects which may be indistinguishable from other tumours in the immediate vicinity, i.e. in the postero-superior part of the third ventricle and the upper part of the brain stem. The mechanical effect of such tumours is to produce hydrocephalus from obstruction to the circulation of the cerebrospinal fluid in the posterior part of the third ventricle and aqueduct of Sylvius. Thus, there is usually headache, vomiting, stupor and papilloedema. The neurological abnormalities are often limited to the oculomotor mechanism and are characterized by defective upward movement of the eyeballs and immobility of the pupils to stimulation by light, with preservation of the reaction on accommodation and convergence. There is often a degree of 


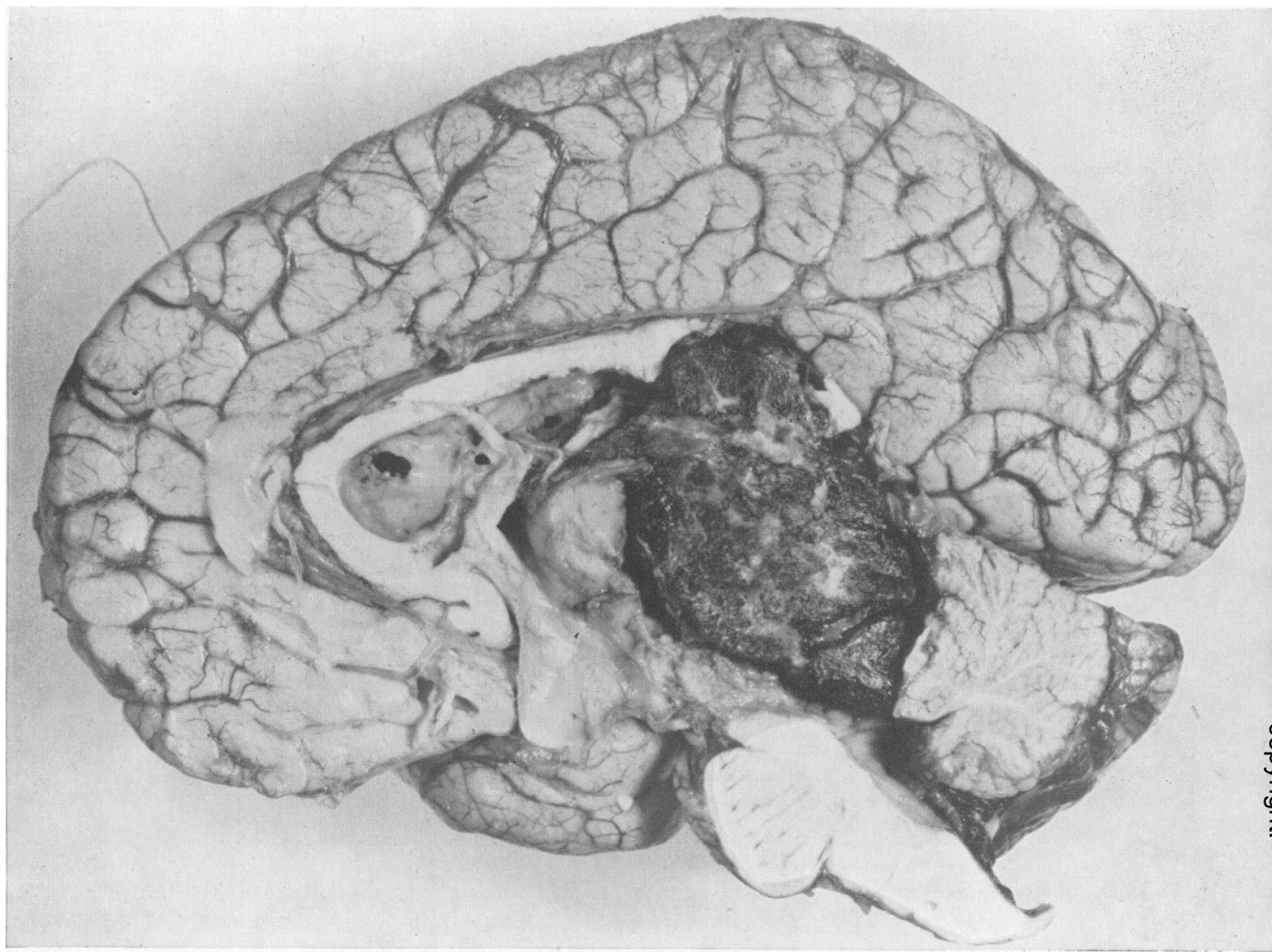

FIG. I6.-Pineal tumour.

bilateral ptosis and there may be a disorder of conjugate lateral movement of the eyeballs akin to skew deviation in which the abducting eye looks outward and downward while the adducting eye looks inward and upward with corresponding diplopia.

In some cases of pineal tumour in boys, there is marked sexual precocity. The more common endocrine effect in males and females is mild hypopituitarism which results from the pressure of a dilated third ventricle on the pituitary gland, a common effect in hydrocephalus due to whatever cause.

The diagnosis of a pineal tumour can sometimes be inferred from the clinical evidence but confirmation depends on ventriculography (Fig. I5). Even so it may be impossible to say whether the characteristic filling defect in the postero-superior part of the third ventricle is due to a pineal tumour or to a tumour arising from neighbouring structures. Attempts at removal carry a high mortality or produce a permanent homonymous hemianopia, $\underline{3}$. and the tendency nowadays is to deal with the hydrocephalus by a short-circuiting procedure such as a ventriculo-cisternostomy or the anterior ventriculostomy described by Stookey and Scarff (in which the anterior wall of the third ventricle, 은 the lamina terminalis, is incised, thus allowing the $\frac{D}{2}$ cerebrospinal fluid to escape from the anterior end of the third ventricle into the subarachnoid space of without having to traverse the posterior end, aqueduct and fourth ventricle), and to administer radiation treatment afterwards. These measures are often highly successful and we have such cases 2 which have remained free from symptoms for periods up to 14 years. Although not verified histologically, the clinical and ventriculographic evidence left little doubt that there was a tumour in the pineal region, and the response suggests that these may be among the most radio-sensitive tumours in the body. 